\title{
Localization and function of GABA transporters GAT-1 and GAT-3 in the basal ganglia
}

\section{Xiao-Tao Jin* , Adriana Galvan, Thomas Wichmann and Yoland Smith}

Division of Neuroscience, Yerkes National Primate Research Center and Department of Neurology, Emory University, Atlanta, GA, USA

\section{Edited by:}

James M. Tepper, Rutgers - The State

University of New Jersey, USA

Reviewed by:

Atsushi Nambu, Graduate University

for Advanced Studies, Japan

Hitoshi Kita, The University of

Tennessee Health Science Center.

USA

\section{*Correspondence}

Xiao-Tao Jin, Division of Neuroscience Yerkes National Primate Research Center and Department of Neurology, Emory University, 954 Gatewood Road NE, Atlanta, GA 30322, USA. e-mail: jxiaota@emory.edu
GABA transporter type 1 and 3 (GAT-1 and GAT-3, respectively) are the two main subtypes of GATs responsible for the regulation of extracellular GABA levels in the central nervous system. These transporters are widely expressed in neuronal (mainly GAT-1) and glial (mainly GAT-3) elements throughout the brain, but most data obtained so far relate to their role in the regulation of GABA receptor-mediated postsynaptic tonic and phasic inhibition in the hippocampus, cerebral cortex and cerebellum. Taking into consideration the key role of GABAergic transmission within basal ganglia networks, and the importance for these systems to be properly balanced to mediate normal basal ganglia function, we analyzed in detail the localization and function of GAT-1 and GAT-3 in the globus pallidus of normal and Parkinsonian animals, in order to further understand the substrate and possible mechanisms by which GABA transporters may regulate basal ganglia outflow, and may become relevant targets for new therapeutic approaches for the treatment of basal ganglia-related disorders. In this review, we describe the general features of GATs in the basal ganglia, and give a detailed account of recent evidence that GAT-1 and GAT-3 regulation can have a major impact on the firing rate and pattern of basal ganglia neurons through pre-and post-synaptic $\mathrm{GABA}_{A^{-}}$and $\mathrm{GABA}_{B}$-receptor-mediated effects.

Keywords: GABA transporter, striatum, globus pallidus, substantia nigra, patch clamp recording

\section{INTRODUCTION}

GABA is the main neurotransmitter used in the basal ganglia network, and abnormal transmission at specific GABAergic synapses underlies some of the pathophysiological features of various basal ganglia diseases. A tight regulation of GABA homeostasis is essential to mediate normal basal ganglia functions. In this manuscript, we will provide a brief overview of the main characteristics of the different subtypes of GABA transporters in the mammalian CNS, and then discuss some of our recent findings and those from other laboratories about the localization and functions of GABA transporters (GATs) in the basal ganglia. This review does not intend to cover the extensive literature on GATs, but will specifically focus on the distribution and regulatory mechanisms by which these transporters modulate neuronal activity and synaptic transmission in the basal ganglia. Because of the limited amount of data available, this review does not aim at generating integrative concepts about GATs function in the basal ganglia. It is rather focused on the presentation of recent findings that have been gathered about these transporters in specific basal ganglia nuclei, and their potential importance for basal ganglia function and dysfunction. For a more comprehensive account of our current knowledge of GAT function in other brain regions, readers are referred to previous reviews (Borden, 1996; Gadea and Lopez-Colome, 2001; Dalby, 2003; Conti et al., 2004).

\section{GENERAL FEATURES OF GABA TRANSPORTERS}

GABA is the main inhibitory neurotransmitter in the mammalian brain. After release from presynaptic terminals, GABA is rapidly removed from the extracellular space by GATs, a regulatory mechanism that terminates inhibitory synaptic transmission (Borden,
1996; Richerson and $\mathrm{Wu}, 2003)$, regulates GABA spillover to neighboring synapses (Borden, 1996; Overstreet and Westbrook, 2003), and maintains GABA homeostasis to prevent excessive tonic activation of synaptic and extrasynaptic GABA receptors (Borden, 1996; Semyanov et al., 2004). In addition, the reversal of the GATs function may result in additional GABA release under certain pathological and physiological conditions (Allen et al., 2004; Wu et al., 2007).

\section{GATS CLONING AND PHARMACOLOGY}

To date, four different GATs have been described, GAT-1, GAT-2, GAT-3, and the Betain/GABA transporter type 1 (BGT-1). These transporters are members of a large family of 12-transmembrane spanning $\mathrm{Na}^{+} / \mathrm{Cl}^{-}$coupled transporters (for review, see Borden, 1996). GAT-1 was the first GAT to be cloned (Guastella et al., 1990). The GAT-1 protein sequence in rat (Guastella et al., 1990), mouse (Liu et al., 1993), and human (Nelson et al., 1990) displays a high degree of homology and nearly identical pharmacological properties (Borden, 1996). The GAT-2 and GAT-3, cloned by Borden et al. (1992), display a higher degree of amino acid identity between each other (67\% identity), and with the fourth GABA transporter, BGT-1 (68 and 65\% identity for GAT-2 and GAT-3, respectively) than with GAT-1 ( $\sim 52 \%$ amino acid identity). The amino acid sequence of GAT-3 in human, rat, and mouse is virtually identical with only a few substitutions (for review, see Borden, 1996). In contrast to other transporters, BGT-1, cloned by Yamauchi et al. (1992), utilizes both GABA and betaine as substrates.

GATs exchange GABA for $\mathrm{Na}^{+}$and $\mathrm{Cl}^{-}$. The GABA-transporting function of GATs is particularly dependent on the $\mathrm{Na}^{+}$gradient across the membrane. Although $\mathrm{Cl}^{-}$can significantly enhance the 
rate of transport, $\mathrm{Cl}^{-}$alone does not drive GABA uptake in the absence of $\mathrm{Na}^{+}$. The proposed stoichiometry for GAT-1, GAT-2, and GAT-3 is $2 \mathrm{Na}^{+}: 1 \mathrm{Cl}^{-}: 1$ GABA (Loo et al., 2000; Sacher et al., 2002; Karakossian et al., 2005).

GAT-1 can be pharmacologically isolated from GAT-2, GAT-3, and BGT-1. Various drugs have been identified as highly specific GAT-1 inhibitors (for instance, Cl966, SKF 89976A, NO-711, and Tiagabine), while SNAP 5114 is a semiselective blocker of GAT-2 and GAT-3, with a higher affinity for GAT-3 than GAT-2 (IC50 $\sim 5$ and $20 \mu \mathrm{M}$, respectively). However, because GAT-3 is far more abundant in neurons and glia than GAT-2, SNAP 5114 is commonly used as a GAT-3 blocker in studies of GATs regulation of synaptic transmission in the central nervous system. Microdialysis experiments in rodent hippocampus and thalamus have shown that either local or systemic application of GAT-1 antagonists can increase extracellular GABA concentrations by up to 1.5- to 4-folds the basal levels (Richards and Bowery, 1996; Dalby, 2000). Similarly, application of the GAT-2/GAT-3 blocker, SNAP 5114 $(100 \mu \mathrm{M})$, increases GABA levels in the thalamus by almost $250 \%$, but has no significant effect on hippocampal GABA concentration (Dalby, 2000).

\section{GATS LOCALIZATION IN THE CNS}

The cellular localization of GABA transporters has been studied in the rat brain using both in situ hybridization for mRNA (Rattray and Priestley, 1993; Brecha and Weigmann, 1994; Augood et al., 1995; Durkin et al., 1995; Jursky and Nelson, 1996; Nishimura et al., 1997; Yasumi et al., 1997; Ficková et al., 1999) and immunocytochemistry for transporters protein (Ikegaki et al., 1994; Augood et al., 1995; Minelli et al., 1995; Itouji et al., 1996; Ribak et al., 1996; Conti et al., 1998). The GAT-1 mRNA is expressed throughout the brain, but particularly enriched in the olfactory bulb, basal ganglia, interpeduncular nucleus, cerebellum, and retina (Augood et al., 1995; Durkin et al., 1995; Yasumi et al., 1997). Immunohistochemical studies using antibodies raised against recombinant proteins have shown that GAT-1 is not only expressed in GABAergic neurons, but also in non-GABAergic cells and glia in certain brain regions (for review, see Eulenburg and Gomeza, 2010), although their function in these neurons remains poorly understood.

GAT-2 mRNA is weakly expressed throughout the brain, primarily in arachnoid and ependymal cells, and to a much lesser extent, in neurons and astrocytes (Durkin et al., 1995; Conti et al., 1999). GAT-3 mRNA and protein are found predominantly in glial cells (Radian et al., 1990; Ikegaki et al., 1994; Durkin et al., 1995). The strongest GAT-3 expression is found in the glomerular layer of the olfactory bulb, the inner nucleus of the retina, the thalamic paraventricular nucleus, and the globus pallidus (GP; Clark et al., 1992; Ikegaki et al., 1994; Durkin et al., 1995; Minelli et al., 1996). Some of these studies showed that GAT-3 is nearly absent from the neocortex and cerebellar cortex, and very weakly expressed in the hippocampus (Clark et al., 1992; Brecha and Weigmann, 1994; Ikegaki et al., 1994; Durkin et al., 1995), while others provided evidence for significant neocortical expression in rodents (Minelli et al., 1996, 2003; Pow et al., 2005). Finally, low to moderate levels of BGT-1 are expressed in most brain regions (Durkin et al., 1995; Zhou and Ong, 2004).

\section{GATS REGULATION OF SYNAPTIC TRANSMISSION AND PLASTICITY}

The effects of GAT-1 modulation on synaptic transmission have been most studied in the CNS. A summary of the main effects of GAT blockade on GABA release and postsynaptic currents in various CNS regions is shown in Table 1. GAT-1 inhibitors increase the decay of evoked IPSCs, while not having significant effects on IPSC amplitude in many brain regions (Roepstorff and Lambert, 1992; Thompson and Gähwiler, 1992; Engel et al., 1998; Overstreet and Westbrook, 2003). GAT-1 inhibitors also increase GABA receptormediated tonic conductances in cerebellar granule cells (Rossi et al., 2003) as well as in granule cells and pyramidal neurons of the hippocampal dentate gyrus (Nusser and Mody, 2002; Semyanov et al., 2003; Sipilä et al., 2007). A recent study also demonstrated that GAT-1 blockade or genetic deletion of GAT-1 specifically impairs long-term potentiation (LTP) induced by theta burst stimulation (Gong et al., 2009) in the CA1 region of mouse hippocampus. While there is compelling evidence that GAT-1 regulates GABAergic transmission in the hippocampus (Thompson and Gähwiler, 1992; Isaacson et al., 1993; Draguhn and Heinemann, 1996; Engel et al., 1998; Nusser and Mody, 2002; Overstreet and Westbrook, 2003; Semyanov et al., 2003), cerebral cortex (Keros and Hablitz, 2005; Bragina et al., 2008; Gonzalez-Burgos et al., 2009), and cerebellum (Rossi et al., 2003), much less is known about the functional role of GAT-1 in the basal ganglia (Rossi et al., 2003; Galvan et al., 2005; Kinney, 2005; Kirmse et al., 2009). Despite its widespread and abundant expression in many brain regions (see Borden, 1996; Eulenburg and Gomeza, 2010 for reviews), the role of GAT-3-mediated regulation of GABAergic transmission remains poorly understood compared with GAT-1 functions in most CNS regions, except for the cerebral cortex and some basal ganglia nuclei (Table 1).

\section{GAT-1 AND GAT-3 IN THE BASAL GANGLIA}

The relative importance of GAT-1 and GAT-3 in the normal and pathological functioning of the basal ganglia, and the possibility that their regulation could be used to achieve beneficial therapeutic responses in basal ganglia disorders remain largely unexplored. In the following sections, we describe the current knowledge of the localization and function of GAT-1 and GAT-3 in various basal ganglia nuclei, and critically discuss their potential relevance as targets for drug therapies of basal ganglia disorders, such as Parkinson's disease.

\section{STRIATUM}

\section{GAT-1 AND GAT-3 LOCALIZATION}

Most neurons in the striatum, including medium spiny projection neurons and several interneuron subtypes are GABAergic (Kawaguchi et al., 1990). The maintenance of homeostasis in extracellular levels of GABA and GABAergic transmission is, therefore, critical for normal striatal functions. Although the chemical phenotype of most striatal GAT-1-positive cells remains to be determined, it is clear that a significant proportion of the GABAergic neurons in the striatum (including medium spiny neurons and a large proportion of parvalbumin-positive interneurons), express mRNA and immunoreactivity for GAT-1 (Augood et al., 1995; Durkin et al., 1995; Yasumi et al., 1997; Wang and Ong, 1999).

In contrast, the evidence for striatal GAT-3 expression remains controversial. Some of the existing in situ hybridization studies have reported negative data (Clark et al., 1992; Durkin et al., 1995), 


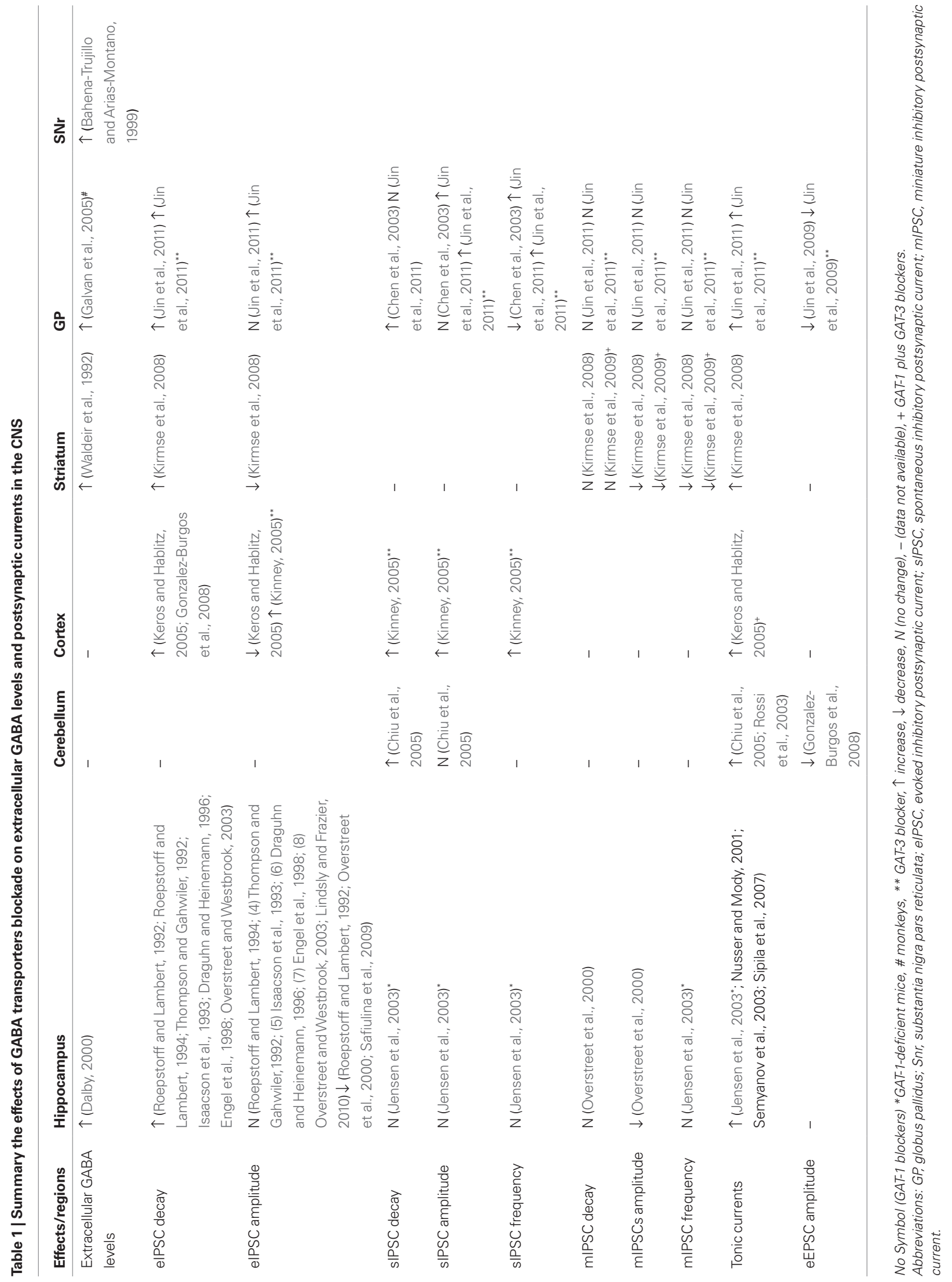


while others using similar methods or polymerase chain reaction (rt-PCR) techniques have demonstrated a significant level of striatal GAT-3 mRNA expression in the rat caudate-putamen (Yasumi et al., 1997; Ficková et al., 1999). GAT-3 immunoreactivity was also demonstrated in the monkey striatum (Ng et al., 2000). The cellular and chemical phenotypes of GAT-3-positive striatal elements remain poorly characterized in both primates and non-primates.

\section{FUNCTIONAL ROLE OF STRIATAL GAT-1}

Systemic or local application of the selective GAT-1 inhibitor, SKF 89976A, doubles the extracellular concentration of GABA in the rat striatum (Waldmeier et al., 1992). Consistent with this finding, GAT-1 blockade induces $\mathrm{GABA}_{\mathrm{A}}$ receptor-mediated tonic inhibition of striatal neurons (Kirmse et al., 2008), similar to previous reports in the hippocampus (Jensen et al., 2003; Semyanov et al., 2003; Scimemi et al., 2005) and cerebellar Purkinje cells (Chiu et al., 2005). Electrophysiologic brain slice recording studies have demonstrated that bath application of the GAT-1 inhibitors NO-711 prolongs the decay time of IPSCs evoked locally in striatum (Kirmse et al., 2008) and decreases the amplitude of eIPSCs produced by intrastriatal stimulation. The latter effect is most likely caused by a presynaptic mechanism because it was associated with a significant increase of the paired-pulse facilitation ratio (PPR; Figures 1A,B). Interestingly, coapplication of NO-711 and the $\mathrm{GABA}_{\mathrm{B}}$-receptor antagonist CGP55845 only partly restored the GAT-1 blockademediated effects on the amplitude of eIPSCs but reduced the PPR to control levels (Figures 1C-E), suggesting that the effects of GAT-1 blockade upon eIPSCs are partially, but not fully mediated by $\mathrm{GABA}_{\mathrm{B}}$ receptor-dependent mechanisms (Kirmse et al., 2008). This observation was recently extended to the hippocampus (Safiulina and Cherubini, 2009; Lindsly and Fraxier, 2010). Other mechanisms, including postsynaptic shunting and $\mathrm{GABA}_{\mathrm{A}}$-receptor desensitization due to a persistent activation of $\mathrm{GABA}_{\mathrm{A}}$ receptors by high ambient GABA concentration in the presence of NO-711 could also contribute to these effects (Overstreet et al., 2000; Keros and Hablitz, 2005; Kirmse et al., 2008).

GATs, acting in reverse direction, also contribute to the action potential-independent release of GABA in the rat striatum (Bernath and Zigmond, 1989; Del Arco et al., 1998; Schoffelmeer et al., 2000). For instance, microdialysis studies have shown that nipecotic acid, a non-selective GAT blocker, attenuates the amphetamine-induced increase in extracellular concentration of GABA in the striatum of freely moving rats, using a calcium-free microdialysis medium (Del Arco et al., 1998). Nipecotic acid also attenuates glutamate NMDA and dopamine D1-like receptor-mediated $\left[{ }^{3} \mathrm{H}\right]$-GABA release from striatal slice and cultured striatal neurons in the presence of the sodium channel blocker tetrodotoxin (Schoffelmeer et al., 2000). These results demonstrate that the release of GABA induced by amphetamine or activation of D1-like or NMDA receptors involves a GAT mechanism. This reversal of GAT function may play a role in the behaviorally activating effects of psychostimulant drugs (Schoffelmeer et al., 2000).

\section{FUNCTIONAL ROLE OF STRIATAL GAT-3}

Although the anatomical evidence for striatal GAT-3 expression is controversial compared with GAT-1 (Clark et al., 1992; Ficková et al., 1999; Ng et al., 2000), there is functional evidence for an active

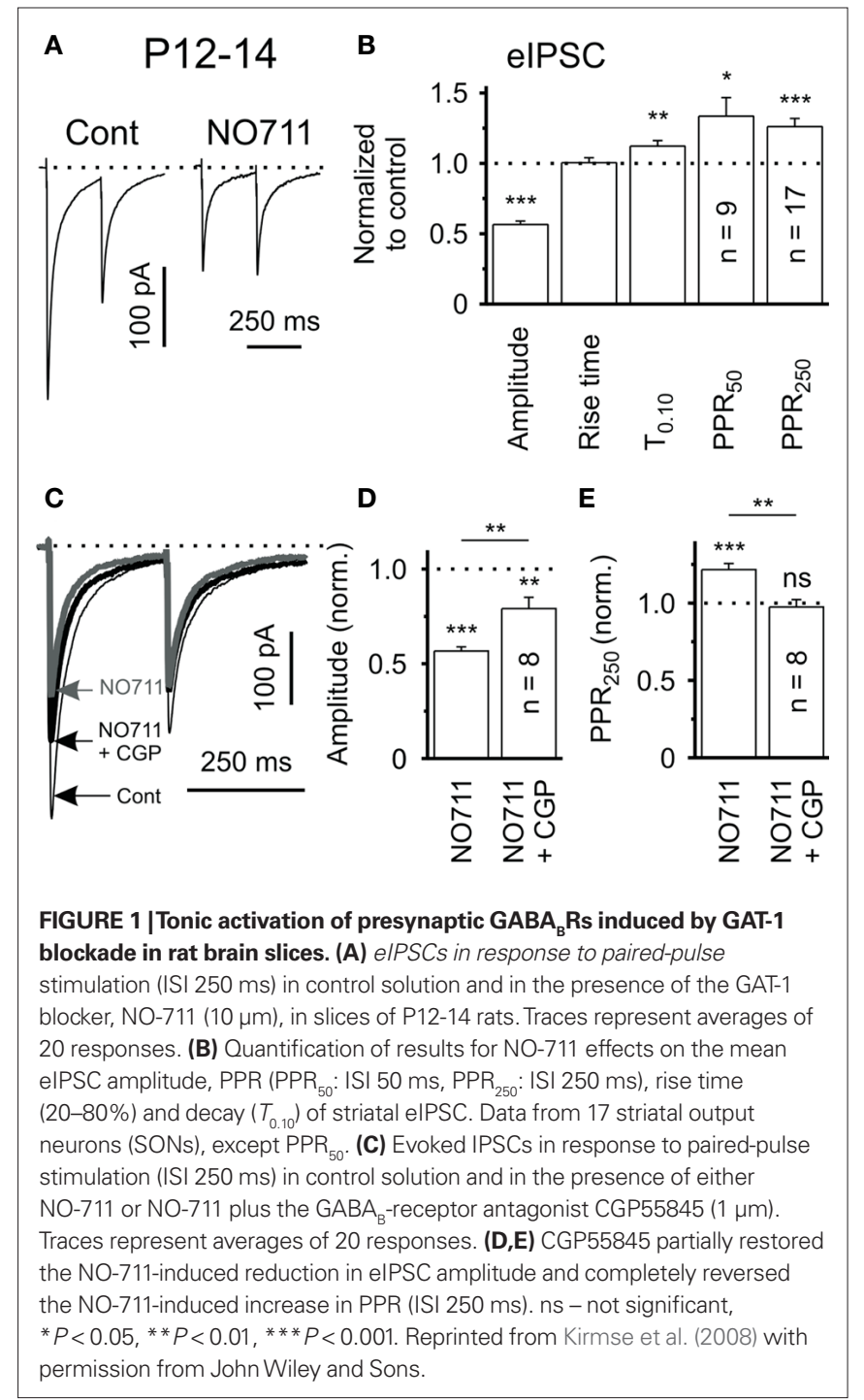

role of GAT-3 in the rat striatum (Kirmse et al., 2009). Despite the fact that the application of SNAP 5114 alone, a semiselective GAT-3 inhibitor, has no effect on striatal GABAergic transmission, and does not affect $\mathrm{GABA}_{\mathrm{A}}$ receptor-mediated tonic currents in striatal projection neurons (Kirmse et al., 2009), the coapplication of SNAP 5114 with the GAT-1 inhibitor NO-711 $(10 \mu \mathrm{M})$ was shown to reduce the frequency of miniature IPSCs (mIPSCs) without affecting their amplitude or kinetic in striatal neurons (Figures 2A-C). As shown for GAT-1, GAT-3 blockade reduces the mean amplitude of eIPSCs through $\mathrm{GABA}_{\mathrm{B}}$ receptor-mediated presynaptic effects in the rat striatum (Kirmse et al., 2009). As the functional effects of GAT-3 blockade on GABAergic transmission may be masked by concomitant GAT-1-mediated effects, it is possible that GAT-3-mediated GABA uptake comes into play only under specific physiological or pathological conditions that result in increases in neuronal activity and GABA release beyond normal levels in the striatum (Kirmse et al., 2009). In other brain regions, it has been shown that that GAT-1 and GAT-3 are largely segregated in pre-terminal neuronal elements and glia, respectively, thus, it 


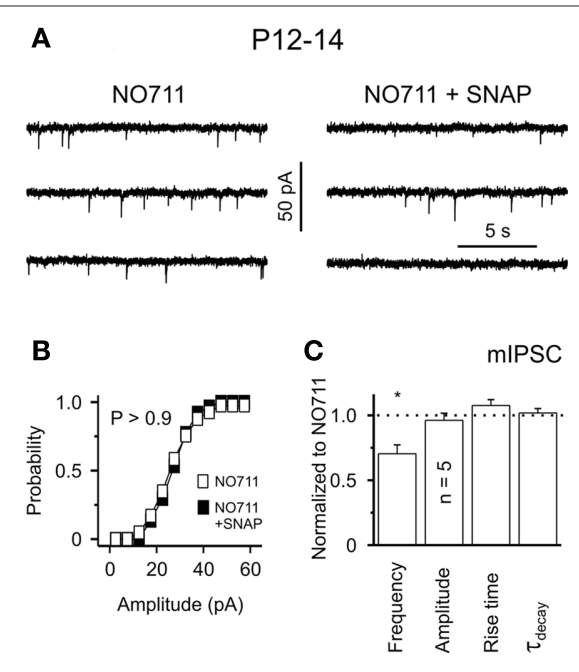

FIGURE 2 | Functional expression of GAT-2/3 is unmasked by suppression of GAT-1 activity in the rat striatum. (A) Sample traces showing mIPSCs in the presence of NO-711 $(10 \mu \mathrm{M})$ and NO-711 plus SNAP $5114(40 \mu \mathrm{M})$ in striatal slices of $\mathrm{P} 12-14$ rats. (B) Cumulative mIPSCs amplitude distribution was not significantly affected by SNAP $5114(P>0.9$, Kolmogorov-Smirnov test). Plot represents data from the cell shown in (A). (C) SNAP 5114 selectively decreased the frequency of mIPSCs. ${ }^{*} P<0.05$. Reprinted from Kirmse et al. (2009) with permission from John Wiley and Sons.

is possible that these transporters might play complementary and synergistic roles towards the regulation of GABAergic transmission in the striatum.

\section{GLOBUS PALLIDUS \\ GAT-1 AND GAT-3 LOCALIZATION}

The rat GP expresses strong mRNA for both GAT-1 and GAT-3 (Durkin et al., 1995; Yasumi et al., 1997). Consistent with these findings, our studies have demonstrated strong GAT-1 and GAT-3 immunoreactivity in the rat and monkey GP (Galvan et al., 2005, 2010; Jin et al., 2009, 2011; Figure 3). At the electron microscopic level, GAT-1 is largely expressed in small unmyelinated axons in the rat GP (Figure 3), and in both unmyelinated axons and glial processes in the external and internal segments of the GP (GPe and GPi, respectively) in the monkey (Figure 3). The pattern of GAT-3 immunoreactivity in the rat and monkey pallidum is strikingly different from that of GAT-1, being almost exclusively expressed in glial cell processes which, in some cases (Figure 3) are closely apposed to putative GABAergic terminals forming symmetric synapses or wrapped around axo-dendritic complexes consisting of numerous unlabeled terminals and dendrites of pallidal neurons (Figure 3). Despite significant alterations in GAT function, there is no significant change in the general localization pattern of GAT-1 and GAT-3 in the GPe and GPi of MPTP-treated Parkinsonian monkeys (Figure 3 and below; Galvan et al., 2010).

\section{FUNCTIONAL ROLE OF GAT-1 IN THE GLOBUS PALLIDUS}

In normal monkeys, local intrapallidal administration of the GAT-1 antagonist (SKF 89976A) significantly increases the ambient GABA level in GPe, as measured by microdialysis (Figure 4A), and reduces the firing rate of GPe and GPi neurons (Figures 4B1,B2; Galvan et al.,
2005). We found that the inhibitory effects of GATs blockade on GPi firing are strongly decreased in monkeys rendered Parkinsonians by systemic treatment with the dopaminergic neurotoxin 1-methyl4-phenyl-1,2,3,6-tetrahydropyridine (MPTP), while the effects on GPe discharge rates remain unaffected (Galvan et al., 2010). In line with our monkey data, systemic administration of tiagabine, a GAT-1 antagonist, increases extracellular levels of GABA by up to threefold in the rat GP (Fink-Jensen et al., 1992). Furthermore, bath application of tiagabine has several significant effects when used in rat brain slice recording experiments: (1) GAT-1 inhibition prolongs the decay time of IPSCs evoked by striatal stimulation, without affecting their amplitude (Figures 5A,B). Similar observations have been made in many brain regions, suggesting that it represents a general GAT-1 function in the CNS (Roepstorff and Lambert, 1992; Thompson and Gähwiler, 1992; Engel et al., 1998; Overstreet and Westbrook, 2003; for reviews, see Borden, 1996; Richerson and Wu, 2003). (2) GAT-1 blockade induces $\mathrm{GABA}_{\mathrm{A}}$ receptor-mediated tonic currents in rat GP neurons (Jin et al., 2011), another general role reminiscent of GAT-1-mediated effects in other brain regions (for reviews, see Borden, 1996; Richerson and Wu, 2003; Eulenburg and Gomeza, 2010). (3) The effects of GAT-1 blockade on spontaneous and miniature IPSCs in rat GP are controversial. On one hand, application of tiagabine prolongs the decay kinetics and reduces the frequency of spontaneous and miniature IPSCs, in part through activation of presynaptic $\mathrm{GABA}_{\mathrm{B}}$ autoreceptors (Chen and Yung, 2003). However, we found that the frequency and amplitude of spontaneous, but not miniature, IPSCs is increased following GAT-1 blockade (Jin et al., 2011). The sources of the discrepancy between these different sets of data remain to be established. (4) GAT-1 blockade reduces the frequency, but not the amplitude of mEPSCs (Figures 5E,G), most likely through $\mathrm{GABA}_{\mathrm{B}}$ receptor-mediated presynaptic inhibitory effects upon glutamatergic transmission (Jin and Smith, 2009). Thus, together with data from the striatum, these findings indicate that GAT-1 regulates striatal and pallidal activity through modulation of persistent synaptic $\mathrm{GABA}_{\mathrm{A}}$ receptor-mediated currents (IPSCs), extrasynaptic $\mathrm{GABA}_{\mathrm{A}}$ receptor-mediated tonic currents, and presynaptic GABA $_{B}$ receptors in both GABAergic and glutamatergic terminals (Galvan et al., 2006).

The behavioral effects of GAT-1 blockade in the GP are poorly characterized. Apart from evidence that the unilateral administration of tiagabine in the rat GP induces ipsilateral rotations in rats (Chen and Yung, 2003), very little is known about the influence of GATs on behavior. Given the prominent role of altered GABAergic transmission in parkinsonism (Galvan and Wichmann, 2007), it would be particularly interesting to examine the potential antiparkinsonian effects of GATs. Taking into consideration the physiological effects of GAT-1 blockade on pallidal activity and the proposed pathophysiology of basal ganglia networks in parkinsonism (DeLong, 1990), one could predict that GAT-1 blockade in GPe could induce or exacerbate parkinsonism, due to the increased GABAergic transmission along the indirect pathway, while GAT-1 blockade in GPi could alleviate parkinsonism through increased inhibition of the overactive basal ganglia pallidal outflow to the thalamus and brainstem (DeLong, 1990). However, because we found that the effects of GAT-1 blockers in GPi of Parkinsonian monkeys are altered from normal (Galvan et al., 2010), these speculations are, at best, incomplete with the available data. 


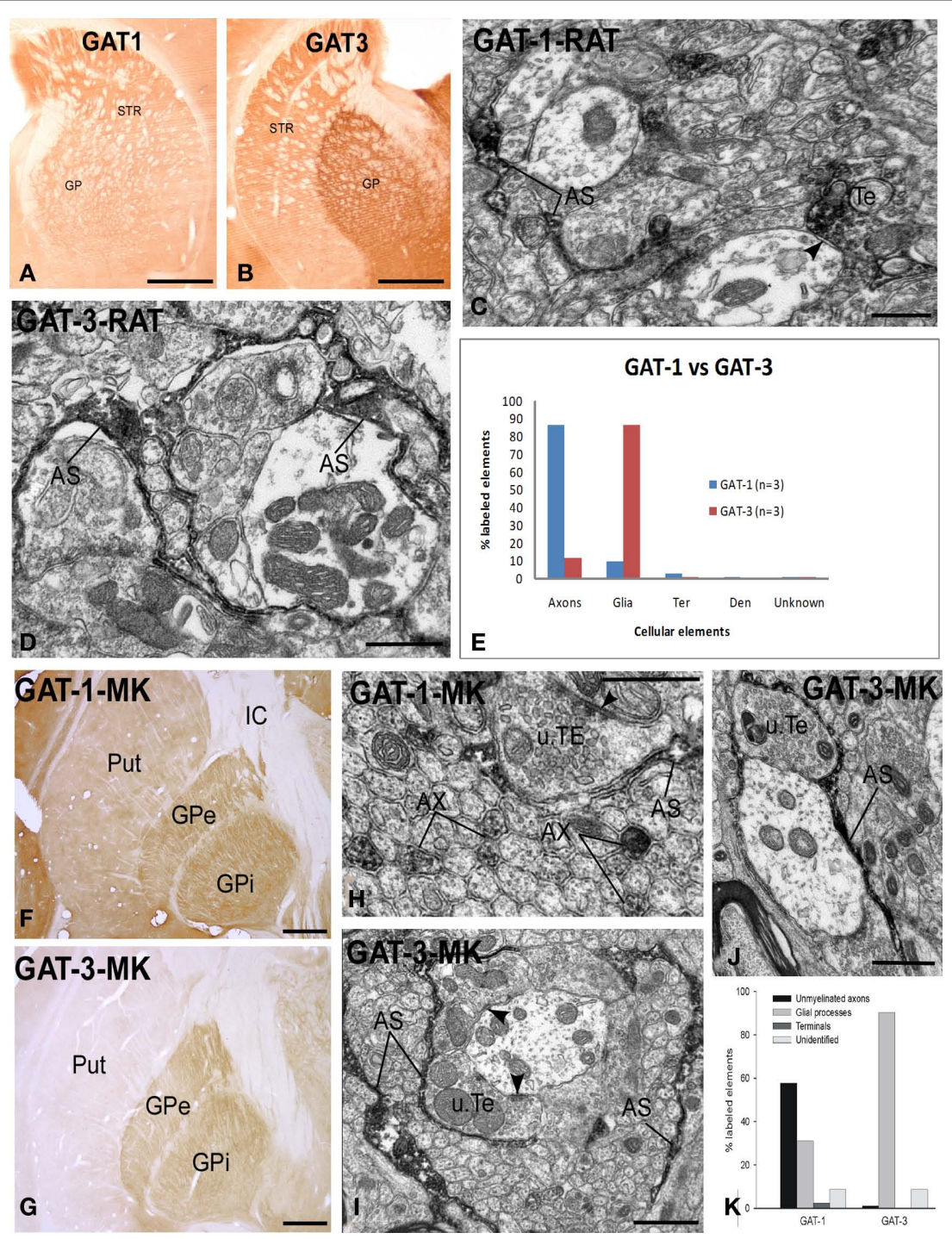

FIGURE 3 | Light and electron micrographs of GAT-1 and GAT-3immunoreactive elements in the rat and monkey globus pallidus.

$(\mathbf{A}, \mathbf{B})$ Light micrographs of GAT-1 and GAT-3 labeling in the rat striatum (STR) and globus pallidus (GP). (C) GAT-1-immunoreactive axon terminal (Te) and astrocytic process (AS) in the rat GP. Note that the labeled terminal forms a symmetric synapse (arrowhead) with an unlabeled dendrite. (D) GAT-3-positive astrocytic processes in close contact with unlabeled terminals and dendrites in the rat GP. (E) Quantification of the percentage of GAT-1- and GAT-3-labeled elements in the rat GP. Note that GAT-1 is predominantly found in unmyelinated axons, whereas GAT-3 is mainly expressed in glial processes. A total of three rats were used in these studies (see Jin et al., 2009, 2011 for details). (F,G) GAT-1 and GAT-3 immunoreactivity in the monkey putamen (Put) and globus pallidus (external and internal segments, GPe, GPi). (H) GAT-1-immunoreactive unmyelinated axons (AX) and astrocytic processes (AS) in the monkey GPe. Note that the labeled AS is in close contact with an unlabeled terminal (u.TE) that forms an axo-dendritic symmetric synapse [arrowhead; (I,J)] GAT-3-positive astrocytes in the monkey GPe (I) and GPi (J). Note the close association between the immunoreactive AS processes and unlabeled axon terminals forming symmetric synapses [arrowheads in (I)]. (K) Quantitative distribution of GAT-1 and GAT-3 across different neuronal and glial elements in the monkey GPe and GPi (see Galvan et al., 2005, 2010 for more details). Scale bars: (A,B,F,G): 1 mm; $(\mathbf{C}, \mathbf{D}, \mathbf{H}, \mathbf{I}, \mathbf{J}): 1 \mu \mathrm{m}$.

\section{FUNCTIONAL ROLE OF GAT-3 IN THE GLOBUS PALLIDUS}

The strong expression of GAT-3 in the pallidum of monkeys and rats suggests that this transporter may play an important role in the clearance of extracellular GABA. Supporting this contention, local in vivo blockade of GAT-3 increases pallidal GABA levels (Figure 4A), and inhibits the firing rate of pallidal neurons in monkeys (Figures $\mathbf{4 C 1 , C 2}$ ). In rat brain slice recording studies, GAT-3 blockade increases the decay and amplitude of evoked IPSCs after striatal stimulation (Figures 5C,D), increases the frequency and amplitude of spontaneous IPSCs, and induces $\mathrm{GABA}_{\mathrm{A}}$ receptor-mediated tonic currents in GP neurons (Jin et al., 2011). The mechanisms by which GAT-3, but not GAT-1, blockade increases the amplitude of evoked IPSCs are unknown. One hypothesis put forward in our recent study (Jin et al., 2011) relates to the fact that GAT-3 blockade may result in the activation of a large pool of striatal GABAergic projections neurons and 

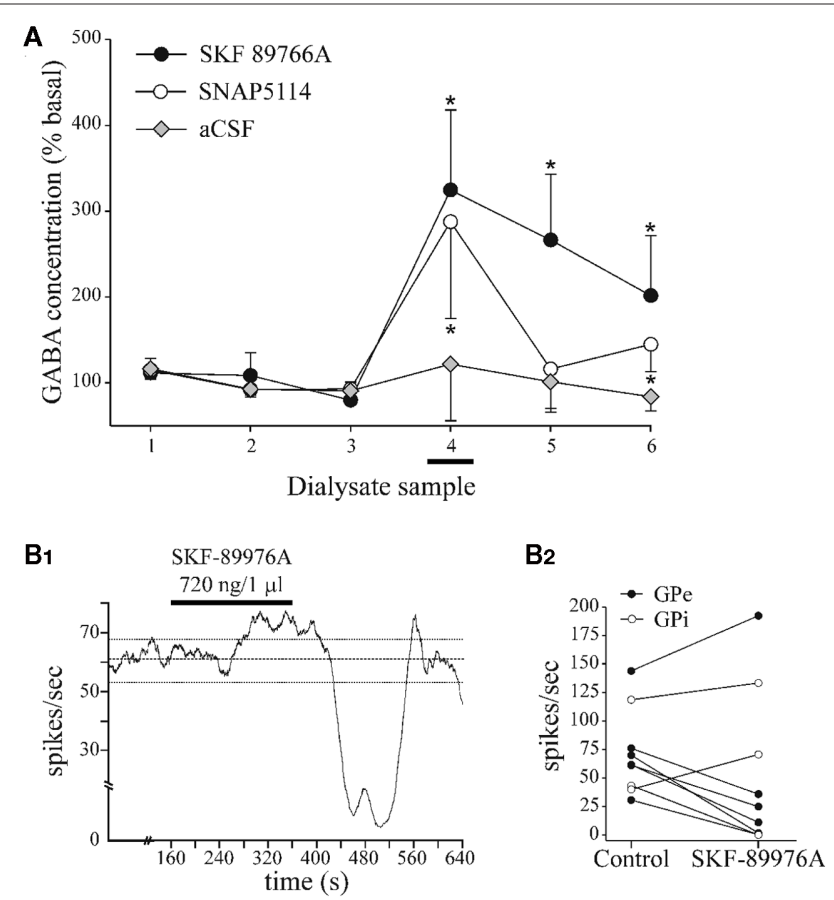

$\mathrm{C}_{1}$

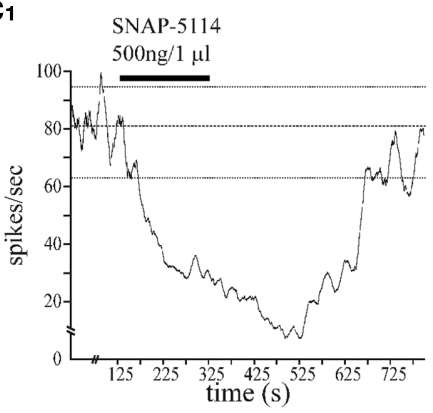

$\mathrm{C}_{2}$

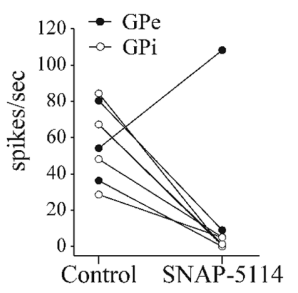

FIGURE 4 | Effects of SKF 89976A and SNAP 5114 on GABA levels in GPe and pallidal discharge rate in monkeys. (A) Dialyzate samples were collected every $10 \mathrm{~min}$. SKF 89976A or SNAP 5114 were administered during sample 4 (horizontal bar). Data area means \pm SD from three experiments for each treatment in a single monkey. Difference from ACSF experiments (MannWhitney UU test): ${ }^{*} P<0.05$. (B1) Example of effect of SKF 89976A on discharge rate of a GPe cell. (B2) Discharge rate of GPe and GPi cells during baseline period and at the point of maximal effect of SKF 89976A. (C1) The discharge rate of this GPe cell is inhibited after administration of the GAT-3 blocker, SNAP 5114. (C2) The discharge rate of GPe and GPi cells during baseline period and at the point of maximal effect of SNAP 5114 injection. In (B1,C1), horizontal bars indicate duration of drug infusions. Dashed lines represent mean discharge rate \pm 2 SD. For more details see Galvan et al. (2005).

interneurons which, under specific conditions, can depolarize other projection neurons, thereby increase GABA release in the GP (Bracci and Panzeri, 2006; Ade et al., 2008).

GAT-3 blockade also reduces glutamatergic transmission, most likely from the subthalamic nucleus, through presynaptic $\mathrm{GABA}_{\mathrm{B}}{ }^{-}$ receptor activation (Figures $\mathbf{5 F}, \mathbf{H}$ ). In contrast with the striatum where the effects of GAT-3 blockade alone are fully masked by GAT-1 (Kirmse et al., 2009), the blockade of GAT-3, alone or in the presence of a GAT-1 blocker, produces significant effects on both
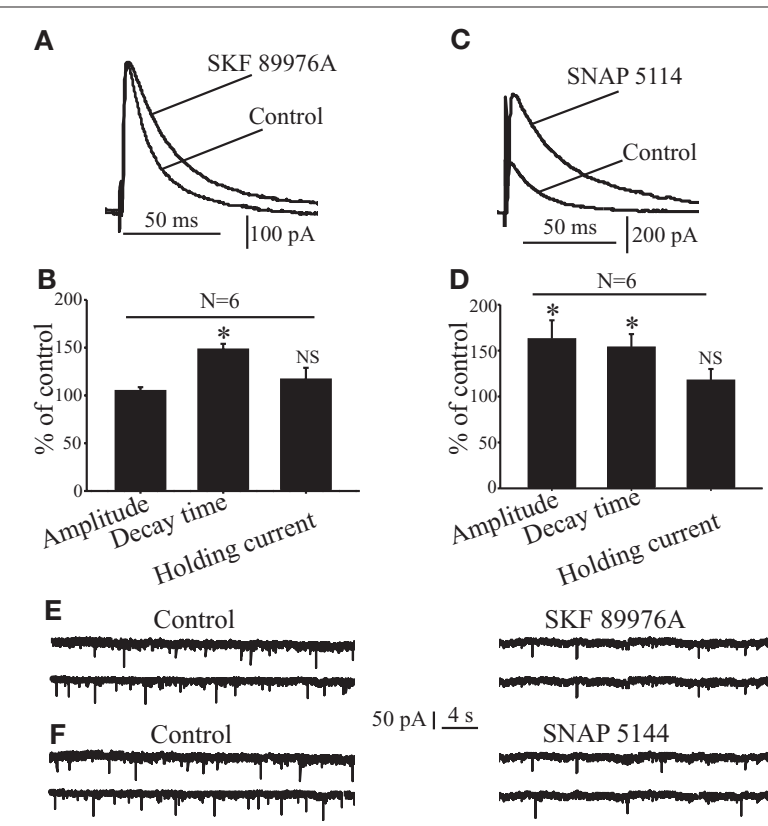

$50 \mathrm{pA} \mid \underline{4 \mathrm{~s}}$
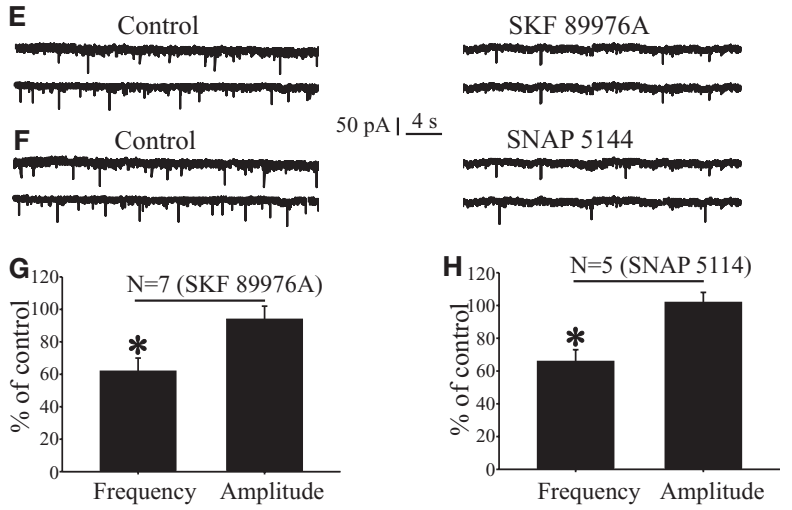

FIGURE 5 | Effects of GAT-1 and GAT-3 blockade on GABAergic and glutamatergic synaptic transmission in the rat GP. (A) Application of SKF 89976 A increases the decay time, but not the amplitude of IPSCs evoked in GP neurons after striatal stimulation. (B) Bar graph showing that SKF 89976A increases the decay time, but has no effect on the amplitude and base line holding currents of elPSCs expressed as percent of control \pm SEM $\left({ }^{*} P<0.005\right)$. (C) Application of SNAP 5114 increases the amplitude and decay time of IPSCs evoked in GP neuron by striatal stimulation. (D) Bar graph summarizing the effects of SNAP 5114 on elPSCs amplitude, decay time, and holding current expressed as percent of control \pm SEM (*P<0.005). (E,F). Sample traces showing mEPSCs recorded in control condition and during SKF 89976A or SNAP 5114 application. (G,H) The summary bar graphs show that SKF 89976A or SNAP 5114 significantly reduce the frequency, but not amplitude of mEPSCs. ${ }^{*} P<0.01$. For more details see Jin et al. $(2009,2011)$.

GABAergic and glutamatergic transmission in the rat GP (Jin et al., 2009, 2011). These differential effects are consistent with localization studies showing that GAT-3 expression in the striatum is comparatively light, compared with the very strong glial expression in the GP (Figure 3; Clark et al., 1992; Durkin et al., 1995; Yasumi et al., 1997; Ng et al., 2000; Galvan et al., 2005; Jin et al., 2009, 2011). GAT-1 and GAT-3, thus, represent differential target sites through which GABA reuptake may subserve a complementary regulation of GABAergic and glutamatergic transmission in the pallidum.

\section{SUBTHALAMIC NUCLEUS AND SUBSTANTIA NIGRA}

Despite their distinct glutamatergic phenotype, rat and human STN neurons, display intense GAT-1 mRNA expression (Yasumi et al., 1997; Augood et al., 1999). There is also evidence from other 
brain regions such as cerebral cortex, inferior colliculus, and the deep cerebellar nuclei, that the number of GAT-1 mRNA-containing cells is much larger than that of GABAergic ( $\mathrm{GAD}_{67}$ mRNA-) positive cells (Swan et al., 1994; Yasumi et al., 1997), suggesting that GAT-1 expression extends beyond GABAergic neurons in these brain regions (Yasumi et al., 1997). The functional significance of GATs in non-GABAergic neurons remains unknown (Augood et al., 1999). However, it is interesting to note that an ongoing gene transfection clinical trial in PD aims at changing the phenotype of subthalamic neurons into GABAergic cells in order to reduce the overactive glutamatergic outflow from the subthalamic nucleus (Lewitt et al., 2011). The expression of GAT-1 into subthalamic terminals may increase the likelihood of success of this approach. As in other basal ganglia nuclei, GAT-3 immunoreactivity is expressed in astrocytic processes throughout the monkey STN (Ng et al., 2000).

The effects of GAT activity on the neuronal activity in the STN are unknown. Because of the preponderance of GABAergic pallidal terminals in the STN, and because of the fact that extrasynaptic GABA-B receptors appear to play important roles in the modulation of burst firing and the pallidosubthalamic "pacemaker" system (Plenz and Kitai, 1999; Bevan et al., 2002), a better understanding of the mechanisms by which GATs modulate GABAergic STN activity is clearly warranted (for review, see Bevan et al., 2007).

Both GAT-1 and GAT-3 mRNAs are moderately expressed in the rat substantia nigra as a whole. Surprisingly, GAT-1 expression is stronger in dopaminergic pars compacta $(\mathrm{SNc})$ neurons than in the GABAergic pars reticulata $(\mathrm{SNr}$ ) cells (Durkin et al., 1995; Yasumi et al., 1997), serving as another possible example of GAT-1-mediated function in non-GABAergic neurons. GAT-3 immunoreactivity is also strongly expressed in the monkey $\mathrm{SN}$, where it appears to be preferentially associated with astrocytes $(\mathrm{Ng}$ et al., 2000). GAT-1 inhibition significantly reduces $\left[{ }^{3} \mathrm{H}\right]$ GABA uptake in synaptosomes prepared from the rat $\mathrm{SNr}$ (Bahena-Trujillo and Arias-Montano, 1999), and significantly increases extracellular GABA levels in the rat substantia nigra (Fink-Jensen et al., 1992). However, the overall regulatory functions of GATs on synaptic transmission in the SN remain unexplored.

\section{GATs AND PARKINSON'S DISEASE}

The data discussed in this review highlight the fact that GAT-1 and GAT-3 represent different target sites through which GABA reuptake may regulate GABAergic and glutamatergic transmission in the

\section{REFERENCES}

Ade, K. K., Janssen, M. J., Ortinski, P. I., and Vivini, S. (2008). Differential tonic GABA conductances in striatal medium spiny neurons. J. Neurosci. 28, 1185-1197.

Allen, N. J., Karadottir, R., and Attwell, D. (2004). Reversal or reduction of glutamate and GABA transport in CNS pathology and therapy. Eur. J. Physiol. 449, 132-142.

Augood, S. J., Herbison, A. E., and Emson, P. C. (1995). Localization of GAT-1 GABA transporter mRNA in rat striatum: cellular coexpression with

basal ganglia. Although still poorly understood, recent in vitro and in vivo data from our laboratory and others have demonstrated that GAT-1 and GAT-3 are strongly expressed in the GP, and that their blockade significantly impacts the activity of pallidal neurons under normal and Parkinsonian conditions, by increasing GABA levels and subsequent overactivation of $\mathrm{GABA}_{\mathrm{A}}$ - and $\mathrm{GABA}_{\mathrm{B}}$-receptors (Galvan et al., 2005, 2010; Jin et al., 2009, 2011).

Our recent data from primate experiments demonstrate that the impact of GAT-1 and GAT-3 blockade upon neuronal activity is reduced in the GPi of Parkinsonian animals compared with the normal state, despite the fact that the overall pattern of GAT distribution does not change (Galvan et al., 2010). The possible use of drugs that regulate GAT function to treat patients with Parkinson's disease remains speculative. The essential need of tightly regulated GABA homeostasis for normal brain functions reduces the likelihood that systemic administration of GAT blockers could be done without the risk of significant side effects, although such an approach has shown some therapeutic benefits in patients with epilepsy and anxiety (Gadea and Lopez-Colome, 2001; Dalby, 2003; Sarup et al., 2003; Conti et al., 2004; Schwartz and Nihalani, 2006).

Recent evidence suggests that the interaction of GATs with other receptor systems may provide an alternative strategy for the development of Parkinson's disease therapy. For instance, a recent study has demonstrated that adenosine inhibits GAT-1-mediated GABA uptake in the rat GP (Gonzalez et al., 2006). This finding, combined with the fact that A2A-receptor antagonists have significant antiparkinsonian effects (Kanda et al., 2000; Chase et al., 2003), raise the possibility that the antiparkinsonian effects of A2Areceptor antagonists are partly due to presynaptic modulation of GABA release at striatopallidal synapses through disinhibition of GAT-1 function. GABA uptake is also modulated by activation of cannabinoid CB1 receptors in the rat GP (Venderova et al., 2005), providing another mechanism that could be used to regulate the overactive GABAergic striatopallidal transmission in Parkinson's disease (Romero et al., 2002; Brotchie, 2003). These mechanisms of indirect modulation of GAT activity may represent a more promising therapeutic strategy in the treatment of Parkinson's disease than use of primary GAT blockers (for review, see Conti et al., 2004).

\section{ACKNOWLEDGMENTS}

This research was supported by NIH grant RO1 NS 0432937 and the Yerkes Primate Center NIH base Grant (RR-00165). Thanks to Jean-Francois Pare and Xing Hu for technical assistance.

phorbol ester-induced inhibition. Neurosci. Lett. 274, 119-122.

Bernath, S., and Zigmond, M. J. (1989). Dopamine may influence striatal GABA release via three separate mechanisms. Brain Res. 476, 373-376.

Bevan, M. D., Hallworth, N. E., and Baufreton, J. (2007). GABAergic control of the subthalamic nucleus. Prog. Brain Res. 160, 173-188.

Bevan,M.D., Magill,P.)., Terman,D., Bolam, J. P., and Wilson, C. J. (2002). Move to the rhythm: oscillations in the subthalamic nucleus-external globus pallidus network. Trends Neurosci. 25, 525-531.
Borden, L. A. (1996). GABA transporter heterogeneity: pharmacology and cellular localization. Neurochem. Int. 29, 335-356.

Borden, L. A., Smith, K. E., Hartig, P. R., Branchek, T. A., and Weinshank, R. L. (1992). Molecular heterogeneity of the $\gamma$-aminobutyric acid (GABA) transport system. J. Biol. Chem. 267,21098-21104.

Bracci, E., and Panzeri, S. (2006). Excitatory GABAergic effects in striatal projection neurons. J. Neurophysiol. 95, 1285-1290.

Bragina, L., Marchionni, I., Omrani, A., Cozzi, A., Pellegrini-Giampietro, D. 
E., Cherubini, E., and Conti, F. (2008). GAT-1 regulates both tonic and phasic GABAA receptor-mediated inhibition in the cerebral cortex. J. Neurochem. 105, 1781-1793.

Brecha, N. C., and Weigmann, C. (1994). Expression of GAT-1, a high-affinity gamma-aminobutyric acid plasma membrane transporter in the rat retina. J. Comp. Neurol. 345, 602-611.

Brotchie, J. M. (2003). CB1 cannabinoid receptor signalling in Parkinson's disease. Curr. Opin. Pharmacol. 3, 54-61.

Chase, T. N., Bibbiani, F., Bara-Jimenez, W., Dimitrova, T., and Oh-Lee, J. D. (2003). Translating A2A antagonist KW6002 from animal models to Parkinsonian patients. Neurology 61, S107-S111.

Chen, L., and Yung, W. H. (2003). Effects of the GABA-uptake inhibitor tiagabine in rat globus pallidus. Exp. Brain Res. 152, 263-269.

Chiu, C. S., Brickley, S., Jensen, K., Sputhwell, A., Mckinney, S., Candy, S. C., Mody, I., and Laster, H. A. (2005). GABA transporter deficiency causes tremor, ataxia, nervousness, and increased GABA induced tonic conductance in cerellum. J. Neurosci. 25 , 3234-3245.

Clark, J. A., Deutch, A. Y., Gallipoli, P. Z., and Amara, S. G. (1992). Functional expression and CAN distribution of a $\beta$-alanine-sensitive neuronal GABA transporter. Neuron 9, 337-348.

Conti, F., Melone, M., De Biasi, S., Minelli, A., Brecha, N. C., and Ducati, A. (1998). Neuronal and glial localization of GAT-1, a high-affinity gammaaminobutyric acid plasma membrane transporter, in human cerebral cortex: with a note on its distribution in monkey cortex. J. Comp. Neurol. 396, 51-63.

Conti, F., Minelli, A., and Melone, M. (2004). GABA transporters in the mammalian cerebral cortex: localization, development and pathological implications. Brain Res. Rev. 45, 196-212.

Conti, F., Zuccarello, L. V., Barbaresi, P., Minelli, A., Breacha, N. C., and Molone, M. (1999). Neuronal, glial, and epithelial localization of gammaaminobutric acid transporter 2 , a high-affinity gamma-aminobutyric acid plasma membrane transporter, in the cerebral cortex and neighboring structures. J. Comp. Neurol. 409, 482-494.

Dalby, N. O. (2000). GABA-level increasing and anticonvulsant effects of three different GABA uptake inhibitors. Neuropharmacology 39, 2399-2407.

Dalby, N.O. (2003). Inhibition of gammaaminobutyric acid uptake: anatomy, physiology and effects against epilep- tic seizures. Eur. J. Pharmacol. 479, 127-137.

Del Arco, A., Castaneda, T. R., and Mora, F. (1998). Amphetamine releases GABA in the striatum of the freely moving rat: involvement of calcium and high affinity transporter mechanisms. Neuropharmacology 37, 199-205.

DeLong, M. R. (1990). Primate models of movement disorders of basal ganglia origin. Trends Neurosci. 13, 281-285.

Draguhn, A., and Heinemann, U. (1996). Different mechanisms regulate IPSC kinetics in early postnatal and juvenile hippocampal granule cells. J. Neurophysiol. 76, 3983-3993.

Durkin, M. M., Smith, K. E., Borden, L. A., Weinshank, R. L., Branchek, T.A., and Gustafson, E. L. (1995). Localization of messenger RNAs encoding three GABA transporters in rat brain: an in situ hybridization study. Brain Res. Mol. Brain Res. 33, 7-21.

Engel, D., Schmitz, D., Gloveli, T., Frahm, C., Heinemann, U., and Draguhn, A. (1998). Laminar difference in GABA uptake and GAT-1 expression in rat CA1. J. Physiol. 512, 643-649.

Eulenburg, V., and Gomeza, J. (2010). Neurotransmitter transporters expressed in glial cells as regulators of synapse function. Brain Res. Rev. 63, 103-112.

Ficková, M., Dahmen, N., Fehr, C., and Hiemke, C. (1999). Quantitation of GABA transporter 3 (GAT3) mRNA in rat brain by competitive RT-PCR. Brain Res. Protoc. 4, 341-350.

Fink-Jensen, A., Suzdak, P. D., Swedberg, M. D. B., Judge, M. E., Hansen, L., and Nielsen, P. G. (1992). The $\gamma$-amonobutyric acid (GABA) uptake inhibitor, tiagabine, increases extracellular brain levels of GABA in awake rats. Eur. J. Pharmacol. 220, 197-201.

Gadea, A., and Lopez-Colome, A. M. (2001). Glial transporters for glutamate, glycine, and GABA: GABA transporters. J. Neurosci. Res. 63, 461-468.

Galvan, A., Hu, X., Smith, Y., and Wichmann, T. (2010). Localization and function of GABA transporters in the globus pallidus of Parkinsonian monkeys. Exp. Neurol. 223, 505-515.

Galvan, A., Kuwajima, M., and Smith, Y. (2006). Glutamate and GABA receptors and transporters in the basal ganglia: what does their subsynaptic localization reveal about their function? Neuroscience 143, 351-375.

Galvan, A., Villalba, R. M., West, S. M., Maidment, N. T., Ackerson, L. C., Smith, Y., and Wichmann, T. (2005). GABAergic modulation of the activity of globus pallidus neurons in primates: in vivo analysis of the functions of GABA receptors and GABA transporters. J. Neurophysiol. 94, 990-1000.
Galvan, A., and Wichmann, T. (2007). GABAergic circuits in the basal ganglia and movement disorders. Prog. Brain Res. 160, 287-312.

Gong, N., Li, Y., Cai, G. Q., Niu, R. F., Fang, Q., Wu, K., Chen, Z., Lin, L. N., Xu, L., Fei, J., and Xu, T. L. (2009). GABA transporter-1 activity modulates hippocampal theta oscillation and theta burst stimulation-induced long-term potentiation.J. Neurosci. 29, 15836-15845.

Gonzalez, B., Paz, F., Floran, L., Aceves, J., Erlij, D., and Floran, B. (2006). Adenosine $\mathrm{A} 2 \mathrm{~A}$ receptor stimulation decrease GAT-1-mediated GABA uptake in the globus pallidus of the rat. Neuropharmacology 51, 154-159.

Gonzalez-Burgos, G., Rotaru, D. C., Zaitsev, A. V., Povysheva, N. V., and Lewis, D.A. (2009). GABA transporter GAT1 prevents spillover at proximal and distal GABA synapses onto primate preforontal cortex neuron. $J$. Neurophysiol. 101, 533-547.

Guastella, J., Nelson, N., Nelson, H., Czyzyk, L., Keynan, S., Miedel, M. C., Davidson, N., Lester, H. A., and Kanner, B. I. (1990). Cloning and expression of a rat brain GABA transporter. Science 249, 1303-1306.

Ikegaki, N., Saito, N., Hashima, M., and Tanaka, C. (1994). Production of specific antibodies against GABA transporter subtypes (GAT1, GAT2, GAT3) and their application to immunocytochemistry. Mol. Brain Res. 26, 47-54.

Isaacson, J. S., Solis, J. M., and Nicoll, R. A. (1993). Local and diffuse synaptic actions of GABA in the hippocampus. Neuron 10, 165-175.

Itouji, A., Sakai, N., Tanaka, C., and Saito, N. (1996). Neuronal and glial localization of two GABA transporters (GAT1 and GAT3) in the rat cerebellum. Mol. Brain Res. 37, 309-316.

Jensen, K., Chiu, C. S., Sokolova, I., Lester, H.A., and Mody, I. (2003). GABA transporter-1 (GAT-1)-deficient mice: differential tonic activation of GABAA versus GABAB receptors in the hippocampus. J. Neurophysiol. 90, 2690-2701.

Jin, X.-T., Fare, J.-F., and Smith, Y. (2011). Differential localization and functions of GABA transporters, GAT-1 and GAT-3, in the rat globus pallidus. Eur. J. Neurosci. 33, 1504-1518.

Jin, X.-T., Pare, J.-F., and Smith, Y. (2009). "Blockade of GABA transporter (GAT1) modulates the GABAergic transmission in the rat globus pallidus," in Basal Ganglia IX, eds H.J. Groenewegen, H. J. Berendse, and P. J. Voorn (New York: Springer Press), 297-307.

Jin, X.-T., and Smith, Y. (2009). GABA transporters modulate glutamatergic transmission in the rat globus pallidus. Soc. Neurosci. Abstr. 134.18.
Jursky, F., and Nelson, N. (1996). Developmental expression of GABA transporters GAT 1 and GAT4 suggests involvement in brain maturation. $J$. Neurochem. 67, 857-867.

Kanda, T., Jackson, M. J., Smith, L. A., Pearce, R. K., Nakamura, J., Kase, H., Kuwana, Y., and Jenner, P. (2000). Combined use of the adenosine $\mathrm{A}(2 \mathrm{~A})$ antagonist KW-6002 with L-DOPA or with selective $\mathrm{D} 1$ or $\mathrm{D} 2$ dopamine agonist increases antiparkinsonian activity but not dyskinesia in MPTP-treated monkeys. Exp. Neurol. 162, 321-327.

Karakossian, M. H., Spencer, S. R., Gomez, A. Q., Padilla, O. R., Sacher, A., Loo, D. D., Nelson, N., and Eskandari, S. (2005). Novel properties of a mouse gamma-aminobutyric acid transporter (GAT4). J. Membr. Biol. 203, 65-82.

Kawaguchi, Y., Wilson, C. J., and Emson, P. C. (1990). Projection subtypes of rat neostriatal matrix cells revealed by intracellular injection of biocytin. J. Neurosci. 10, 3421-3438.

Keros, S., and Hablitz, J. J. (2005). Subtype-specific GABA transporter antagonists synergistically modulate phasic and tonic GABAA conductances in rat neocortex. J. Neurophysiol. 94, 2073-2085.

Kinney, G. A. (2005). GAT-3 transporters regulate inhibition in the neocortex. J. Neurophysiol. 94, 4533-4537.

Kirmse, K., Dvorzhak, A., Kirischuk, S., and Grantyn, R. (2008). GABA transporter 1 tunes GABAergic synaptic transmission at output neurons of the mouse neostriatum. J. Physiol. 586, 5665-5678.

Kirmse, K., Kirischuk, S., and Grantyn, R. (2009). Role of GABA transporter 3 in GABAergic synaptic transmission at striatal output neurons. Synapse 63, 921-929.

Lewitt, P. A., Rezai, A. R., Leehey, M. A., Ojemann, S. G., Flaherty, A. W., Eskandar, E. N., Kostyk, S. K., Thomas, K., Sarkar, A., Siddigui, M. S., Tatter, S. B., Schwalb, J. M., Poston, K. L., Henderson, J. M., Kurlan, R. M., Richard, I. H., Van Meter, L., Sapan, C. V., During, M. J., Kaplitt, M. G., and Feigin, A. (2011). AAV2-GAD gene therapy for advanced Parkinson's disease: a double-blind, sham-surgery controlled, randomised trial. Lancet Neurol. 10, 309-319.

Lindsly, C., and Fraxier, C. (2010). Two distinct and activity-dependent mechanisms contribute to autoreceptormediated inhibition of GABAergic afferents to hilar mossy cells. J. Physiol. 588, 2801-2822.

Liu, Q.-R., Lopez-Crcuera, B., Mandiyan, S., Nelson, H., and Nelson, N. (1993). Molecular characterization of four pharmacologically distinct 
$\mathrm{y}$-aminobutyric acid transporters in mouse brain. J. Biol. Chem. 268, 2106-2112.

Loo, D. D., Eskandari, S., Boorer, K. J., Sarkar,H.K., and Wright, E.M. (2000). Role of $\mathrm{Cl}$ in electrogenic $\mathrm{Na}$-coupled cotransporters GAT1 and SGLT1. J. Biol. Chem. 275, 37414-37422.

Minelli, A., Barbaresi, P., and Conti, F. (2003). Postnatal development of highaffinity plasma membrane transporters GAT-2 and GAT-3 in the rat cerebral cortex. Dev. Brain Res. 142, 7-18.

Minelli, A., Brecha, N. C., Karschin, C., DeBiasi, S., and Conti, F. (1995). GAT1, a high-affinity GABA plasma membrane transporter, is localized to neurons and astroglia in the cerebral cortex. J. Neurosci. 15, 7734-7746.

Minelli, A., DeBiasi, S., Brecha, N. C., Vitellaro Zuccarello, L., and Conti, F. (1996). GAT-3, a high-affinity GABA plasma membrane transporter, is localized to localized to astrocytic processes, and it is not confined to the vicinty of GABAergic synapses in the cerebral cortex. J. Neurosci. 16, 6255-6264.

Nelson, H., Mandiyan, S., and Nelson, N. (1990). Cloning of the human brain GABA transporter. FEBS Lett. 269, 181-184.

Ng, C. H., Wang, X. S., and Ong, W. Y. (2000). A light and electron microscopic study of the GABA transporter GAT-3 in the monkey basal ganglia and brainstem. J. Neurocytol. 29, 595-603.

Nishimura, M., Sato, K., Mizuno, M., Yoshiya, I., Shimada, S., Saito, N., and Tohyama, M. (1997). Differential expression patterns of GABA transporters (GAT1-3) in the rat olfactory bulb. Mol. Brain Res. 45, 268-274.

Nusser, Z., and Mody, I. (2002). Selective modulation of tonic and phasic inhibitions in dentate gyrus granule cells. J. Neurophysiol. 87, 2624-2628.

Overstreet, L. S., Jones, M. V., and Westbrook, G. L. (2000). Slow desensitization regulates the availability of synaptic GABA(A) receptors. J. Neurosci. 20, 7914-7921.

Overstreet, L. S., and Westbrook, G. L. (2003). Synaptic density regulates independence at unitary inhibitory synapses. J. Neurosci. 23, 2618-2626.

Plenz, D., and Kitai, S. T. (1999). A basal ganglia pacemaker formed by the subthalamic nucleus and external globus pallidus. Nature 400, 677-682.

Pow, D. V., Sullivan, R. K. P., Willliams, S. M., Scott, H. L., Dodd, P. R., and
Finkelstein, D. (2005). Differential expression of the GABA transporters GAT-1 and GAT-3 in brains of rats, cats, monkeys and humans. Cell Tissue. Res. 320, 379-392.

Radian, R., Ottersen, O. P., StormMathisen, J., Castel, M., and Kanner, B. I. (1990). Immunocytochemical localization of the GABA transporter in rat brain. J. Neurosci. 10, 1319-1330.

Rattray, M., and Priestley, J. V. (1993). Differential expression of GABA transporter-1 messenger RNA in subpopulations of GABA neurones. Neurosci. Lett. 156, 163-165.

Ribak, C,.E., Tong, W. M., and Brecha, N. C. (1996). GABA plasma membrane transporters, GAT-1 and GAT-3, display different distributions in the rat hippocampus. J. Comp. Neurol. 367, 595-606.

Richards, D. A., and Bowery, N. C. (1996). Comparative effects of the GABA uptake inhibitors, tiagabine and NNC-711, on extracellular GABA levels in the rat ventrolateral thalamus. Neurochem. Res. 21, 135-140.

Richerson, G. B., and Wu, Y. (2003). Dynamic equilibrium of neurotransmitter transporters: not just for reuptake anymore. J. Neurophysiol. 90, 1363-1374.

Roepstorff, A., and Lambert, J. D. (1992). Comparison of the effect of the GABA uptake blockers, tiagabine and nipecotic acid, on inhibitory synaptic efficacy in hippocampal CAl neurons. Neurosci. Lett. 146, 131-134.

Roepstorff, A., and Lambert, J. D. (1994). Factors contribution to the decay of the stimulus-evoked IPSC in rat hippocampal CA1 neurons. J. Neurophysiol. 72, 2911-2926.

Romero, J., Lastres-Becker, I., de Miguel, R., Berrendero, F., Ramos, J. A., and Fernandez-Ruiz,J. (2002). The endogenous cannabinoid system and the basal ganglia. Biochemical, pharmacological, and therapeutic aspects. Pharmacol. Ther. 95, 137-152.

Rossi, D. L., Hammann, M., and Attwell, D. (2003). Multiple modes of GABAergic inhibition of rat cerebellar cells. $J$. Physiol. 548, 97-110.

Sacher, A., Nelson, N., Ogi, J. T., Wright, E. M., Loo, D. D., and Eskandari, S. (2002). Prestead-state and steadystate kinetics and turnover rate of the mouse gamma-aminobutyric acid transporter (mGAT3) J. Membr. Biol. 190, 57-73.
Safiulina, V. F., and Cherubini, E. (2009). At immature mossy fibers-CA3 connection, activation of presynaptic GABAB receptors bt endogenously released GABA contributes to synapses silencing. Front. Cell. Neurosci. 3:1. doi: 10.3389/neuro.03.001.2009

Sarup, A., Larsson, O. M., and Schousboe, A. (2003). GABA transporters and GABA-transaminase as drug targets. Curr. Drug Targets CNS Neurol. Disord. 2, 369-277.

Schoffelmeer, A. N. M., Vanderschuren, L. J. M. J., Vries, T. J. De., Hogenboom, F., Wardeh, G., and Mulder, A. H. (2000). Synergistically interacting dopamine D1 and NMDA receptors mediate nonvesicular transporter-dependent GABA release from rat striatal medium spiny neurons. J. Neurosci. 20, 3496-3505.

Schwartz, T. L., and Nihalani, N. (2006). Tiagabine in anxiety disorders. Expert Opin. Pharmacother. 7, 1977-1987.

Scimemi, A., Semyanov, A., Sperk, G., Kullmann, D. M., and Walker, M. C. (2005). Multiple and plastic receptors mediate tonic GABAA receptor currents in the hippocampus. J. Neurosci. 25, 10016-10024.

Semyanov, A., Walker, M. C., and Kullmann,D.M. (2003). GABA uptake regulates cortical excitability via cell type-specific tonic inhibition. Nat. Neurosci. 6, 484-490.

Semyanov, A., Walker, M. C., Kullmann, D. M., and Silver, R. A. (2004). Tonically active GABAA receptor: modulating gain and maintaining the tone. Trends Neurosci. 27, 262-269.

Sipilä, S. T., Voipio, J., and Kaila, K (2007). GAT-1 acts to limit a tonic GABAA current in rat CA3 pyramidal neurons at birth. Eur. J. Neurosci. 25, 771-722.

Swan, M., Najelahim, A., Watson, R. E. B., and Benett, J. P. (1994). Distribution of mRNA for the GABA transporter GAT-1 in the rat brain: evidence that GABA uptake is not limited to presynaptic neurons. J. Anat. 185, 315-323.

Thompson, S. M., and Gähwiler, B. H. (1992). Effects of the GABA uptake inhibitor tiagabine on inhibitory synaptic potentials in rat hippocampal slice cultures. J. Neurophysiol. 67, 1698-1701.

Venderova, K., Brown, T. M., and Brotchie, J. M. (2005). Differential effects of endocannabinoids on [3H]-GABA uptake in the rat globus pallidus. Exp. Neuro. 194, 284-287.
Waldmeier, P. C., Stocklin, K., and Feldtrauer, J. J. (1992). Weak effects of local and systemic adaministration of the GABA uptake inhibitor, SKF 89976A, on extracellular GABA in the rat striatum. Naunyn Schmiedebergs Arch. Exp. Pathol. Pharmakol. 345, 544-547.

Wang, X. S., and Ong, W. Y. (1999). A light and electron microscopic study of GAT-1 in the monkey basal ganglia. J. Neurocytol. 28, 1053-1061.

Wu, Y., Wang, W., Diez-Sampedro, A., and Richerson, G. B. (2007). Nonvesicular inhibitory neurotransmission via reversal of the GABA transporter GAT-1. Neuron 56, 851-865.

Yamauchi, A., Uchida, S., Kwon, H. M., Preston, A. S., Robey, R. B., GarciaPerez, A., Burg, M. B., and Handler, J. S. (1992). Cloning of a Na (+)-and $\mathrm{Cl}(-)$-dependent betaine transporter that is regulated by hypertonicity. $J$. Biol. Chem. 267, 649-652.

Yasumi, M., Sato, K., Shimada, S., Nishimura, M., and Tohyama, M. (1997). Regional distribution of GABA transporter 1 (GAT1) mRNA in the rat brain: comparison with glutamic acid decarboxylase67 (GAD67) mRNA localization. Brain Res. Mol. Brain Res. 44, 205-218.

Zhou, X. M., and Ong, W. Y. (2004). A light and electron microscopic study of betaine/GABA transporter distribution in the monkey cerebral neocortex and hippocampus. J. Neurocytol. 33, 233-240.

Conflict of Interest Statement: The authors declare that the research was conducted in the absence of any commercial or financial relationships that could be construed as a potential conflict of interest.

Received: 26 April 2011; paper pending published: 10 May 2011; accepted: 13 July 2011; published online: 28 July 2011. Citation: Jin X-T, Galvan A, Wichmann $T$ and Smith $Y$ (2011) Localization and function of GABA transporters GAT-1 and GAT-3 in the basal ganglia. Front. Syst. Neurosci. 5:63. doi: 10.3389/ fnsys.2011.00063

Copyright (C) 2011 Jin, Galvan, Wichmann and Smith. This is an open-access article subject to a non-exclusive license between the authors and Frontiers Media $S A$, which permits use, distribution and reproduction in other forums, provided the original authors and source are credited and other Frontiers conditions are complied with. 